\title{
Application of biotechnological approaches in genetic and pre-breeding studies of bread wheat
}

\author{
Leonova I.N.*, Kiseleva A.A., Skolotneva E.S., Salina E.A. \\ Institute of Cytology and Genetics, SB RAS, Novosibirsk, Russia \\ *e-mail:leonova@bionet.nsc.ru
}

The development of new forms of common wheat using conventional breeding takes a long period and does not allow to utilize the genetic potential of sources of agronomically important traits. The paper presents the results of the use of biotechnological approaches for screening of wheat varieties on the genetic loci determining valuable traits. To identify QTLs responsible for resistance to fungal pathogens and for yield components, two methodological approaches were used: genome-wide association study (GWAS) and marker-assisted selection. QTL identification was done with help of collection of wheat varieties and mapping populations obtained from hybridization of wheat was cultivars from different groups of ripeness. Loci associated with resistance to fungal diseases were found on chromosomes 5A, 6D, 1D, and 1B. The results of GWAS was confirmed by screening a collection of wheat varieties using STS and SSR markers recommended for marker-assisted selection. Association mapping based on the results of long-term evaluations of wheat varieties on productivity traits revealed 34 loci. For the trait "ear grain number" most SNP are located in the 6th homoeologous group. Loci in chromosome 6A have a positive effect on the trait, whereas QTLs in chromosomes 6B and 6D are negative. Ten significant SNP were found for "ear grain weight". Chromosome 5B has two loci with positive and negative effects. For "1000 grain weight", 14 informative markers were identified in chromosomes 2D, 3A, 4A, 5A, 5B, and 1B. Genetic mapping of the loci for the "ear grain number" and "ear grain weight" was carried out based on the results of trait evaluation in F3 mapping populations. Loci, associated with "ear grain number" were identified in chromosomes $2 \mathrm{D}, 4 \mathrm{~A}, 5 \mathrm{~A}, 5 \mathrm{D}$ and $7 \mathrm{~B}$, with "ear grain weight" - in chromosomes 2D, 3A, 6D and 7A. The obtained results will be used for development of KASP markers associated with yield traits.

Acknowledgements: The research was carried out with the financial support of Russian Science Foundation (grant No. 16-16-00011-P). 